\title{
Artifacts in Musculoskeletal Ultrasonography
}

\author{
Mihra S. Taljanovic, MD, PhD, FACR ${ }^{1} \quad$ David M. Melville, MD ${ }^{1} \quad$ Luke R. Scalcione, MD ${ }^{1}$ \\ Lana H. Gimber, $\mathrm{MD}^{1}$ Eileen J. Lorenz, $\mathrm{MD}^{1}$ Russell S. Witte, PhD ${ }^{1,2}$ \\ ${ }^{1}$ Department of Medical Imaging, the University of Arizona Health \\ Address for correspondence Mihra S. Taljanovic, MD, PhD, FACR, \\ Network \\ 2 Department of Biomedical Engineering, Optical Sciences, University \\ Department of Medical Imaging, the University of Arizona Health \\ Network, 1501 N. Campbell Ave., P.O. Box 245067, Tucson, AZ 85724 \\ of Arizona, Tucson, Arizona \\ (e-mail: mihrat@radiology.arizona.edu).
}

Semin Musculoskelet Radiol 2014;18:3-11.
Abstract
Keywords
- ultrasonography
- ultrasound
- artifact
- anisotropy
- posterior acoustic shadowing
- posterior acoustic enhancement
- increased through transmission
- posterior reverberation
- ring-down artifact
- comet-tail artifact
- mirror image
- beam width artifact
- side-lobe artifact
- background noise
- aliasing
- twinkling artifact

During the past 2 decades, high-resolution ultrasonography (US) has been increasingly utilized in the diagnosis of musculoskeletal trauma and diseases with results comparable with MR imaging. US has an advantage over other cross-sectional modalities in many circumstances due to its superior spatial resolution and ability to allow dynamic assessment. When performing musculoskeletal US, the examiner has to be knowledgeable in the complex anatomy of the musculoskeletal system and US imaging technique. Additionally, he or she must be familiar with several common imaging artifacts in musculoskeletal US that may be mistaken for pathology, as well as several artifacts that frequently accompany pathologic conditions. These artifacts may occur with both Bmode gray-scale and Doppler imaging. In this article, we discuss common artifacts seen in musculoskeletal US and techniques to avoid or minimize these artifacts during clinical US examinations.
High-resolution ultrasonography (US) has proven to be a powerful diagnostic tool in the evaluation of musculoskeletal trauma including injuries of the tendons, ligaments, muscles, and nerves, as well as musculoskeletal diseases including joint effusions, synovitis, bursitis, articular erosions, joint bodies, soft tissue infections, and soft tissue tumors and tumor-like conditions. It can also diagnose radiographically occult fractures and joint and bone infections. ${ }^{1}$ This imaging modality provides results in the diagnosis of musculoskeletal trauma and diseases comparable with MR imaging. ${ }^{2}$

Rapid advances in US technologies in the past 10 years have resulted in significant improvement in image quality. Development of a variety of US transducers, compound imaging, improved focusing, and transducers with large and small fields of view are available for different musculoskeletal applications, resulting in better B-mode gray-scale image quality and information from Doppler imaging. ${ }^{3}$
Issue Theme Variants and Pitfalls in Musculoskeletal Imaging; Guest Editor, Wilfred C.G. Peh, MBBS, MD, FRCP (Glasg), FRCP(Edin), FRCR
Copyright (c) 2014 by Thieme Medical Publishers, Inc., 333 Seventh Avenue, New York, NY 10001, USA. Tel: +1(212) 584-4662.
DOI http://dx.doi.org/ 10.1055/s-0034-1365830. ISSN 1089-7860. 


\section{US Equipment and Technology}

All US machines consist of a transducer (or probe) attached to the machine via a cord. The transducer contains an array of thin piezoelectric crystals, each of which is linked to the machine's electrical system. ${ }^{4}$ The essential component in producing the US image is the piezoelectric crystal, which allows the electrical signal to be converted to ultrasonic waves and vice versa. The US machine sends the electrical signal to the transducer, which results in the production of sound waves. Ultrasound gel (coupling medium) applied to the patient's skin mediates transmission of the US waves into the soft tissues. The sound waves interact with soft tissue interfaces, some of which reflect back to the skin surface and the transducer, where they are converted to electrical current used to create the US image. In the examined tissues that have significantly different acoustic impedance, the sound wave reflection produces a bright echo. Sound waves are reflected more readily by an imaged structure as the angle of insonation approaches 90 degrees, with maximum reflection achieved when the sound wave is directed perpendicular to the imaged structure. In addition to reflection, the sound waves may also be absorbed in the examined tissues, and the amount of absorption increases with greater transducer frequency and tissue viscosity. ${ }^{1}$

An important consideration in US is the range of sound wave frequencies produced by the transducer that significantly affects the image quality. Increasing the transducer frequency improves the spatial resolution at the expense of beam penetration as a result of sound wave absorption. In contrast, lower frequency transducers enable assessment of deeper structures, but the spatial resolution is lower. The transducers may be designed as linear or curvilinear. Linear transducers are ideal for the evaluation of most musculoskeletal structures, with the sound waves propagating in linear form parallel to the transducer surface. Small footprint (hockey stick) linear transducers facilitate examination of the small joints and soft tissue structures about the curved musculoskeletal structures such as tendons and ligaments of the wrist and hand, or ankle and foot. Copious amounts of ultrasound gel may also accommodate the depth and will decrease transducer compression on superficial structures. Curvilinear transducers open the field of view and may be used for evaluation of deeper structures, such as thigh musculature. ${ }^{1}$

In general, accurate adjustment of the settings, precise placement of the focal zones, and appropriate adjustment of penetration depth are all necessary for using the full potentials of current US technology. Depth gain compensation (DGC) allows correction for the depth-related intensity sound drop-off. Although many modern US machines automatically adjust the DGC internally based on the imaging depth, US machines that lack automatic DGC or examiner preference may require manual adjustment of these settings. ${ }^{4}$

\section{Doppler Imaging}

Virtually all machines now offer high-sensitivity spectral, color, and power Doppler imaging, allowing detection of abnormal blood flow in injured or diseased tissues or identification of vessels during interventional procedures. The assessment of Doppler signal in inflammatory or infectious processes enhances interpretation of gray-scale findings, and it may help to evaluate the severity of disease. The presence of the Doppler signal can also help differentiate solid from cystic lesions. In addition, Doppler interrogation enables vascular characterization of solid masses. ${ }^{5,6}$

Doppler shift, which is named after Austrian physicist and mathematician Christian Andreas Doppler who first described it in 1843 as a property of light, ${ }^{7}$ is the change of frequency in a wave when a source moves relative to the receiver. With US imaging, the change of frequency detected between the transmitted and received US frequency is the Doppler shift. ${ }^{5,6}$ Spectral Doppler provides a quantitative analysis of the velocity and direction of the blood flow and refers to the combination of either continuous wave Doppler or pulsed Doppler with a spectral display. In the two-dimensional (2D) spectral display, the frequency shift is depicted on the vertical axis and time on the horizontal axis. The range of blood velocities in the volume produces a corresponding range of frequency shifts. ${ }^{6}$

Color Doppler is an US imaging mode that detects the presence, direction, and velocity of blood flow in a wide range of flow conditions. It provides an estimate of the mean velocity of flow within a vessel by color coding the flow and superimposing it on a 2D gray-scale image. The flow direction is arbitrarily assigned the color red or blue, indicating flow toward or away from the transducer, respectively. Color Doppler US can evaluate a wider area than duplex or power Doppler US, but it is not as precise as conventional duplex Doppler. ${ }^{5-7}$

Power Doppler is the summation of the power amplitude of all the different Doppler shifts (velocities) within a cell that are displayed instead of the velocity average. ${ }^{8}$ Power Doppler uses a different transmit pulse in contrast to color Doppler; it measures the amount (quantity and distribution) of red cells passing by the beam in contrast to the frequency changes of the transmitting and receiving beam. Power Doppler does not indicate flow direction, unlike color Doppler. The intensity of flow is indicated by how close to white the color is on the screen (i.e., yellows are indicative of faster moving blood than reds). Because flow direction is not required, much more sensitive Doppler measurements can be obtained with power Doppler than with color Doppler. Power Doppler US shows improved sensitivity in the detection of low flow in smaller and superficially located vessels, which is of great utility in rheumatologic diseases, tumors, and overuse conditions., However, on present high-end US machines, color Doppler demonstrates comparable sensitivity to power Doppler, and the choice between these two techniques may be made based on actual comparison on the available equipment. ${ }^{7}$

\section{Emerging Technologies}

Most US machines offer spatial compounding, frequency compounding, tissue harmonic imaging, or extended field of view (or panoramic imaging). Tissue harmonic imaging 
improves axial and lateral resolution owing to its higher frequencies and narrower beams, which may increase the conspicuity of normal soft tissue anatomy and abnormalities. In extended field-of-view imaging, the motion of the transducer during a single sweep is tracked, creating a panoramic view of the examined structure. This allows a global picture of the regional anatomy to be obtained and can create images similar to the appearance of MR imaging. ${ }^{9}$

Near-field imaging by the use of higher frequency transducers and harmonic imaging enables improved surface visualization of the examined musculoskeletal structures when compared with conventional B-mode US. With use of spatial compound US, electronic US beam steering from the transducer array during real-time image acquisition results in improved delineation of the lesion or examined structure, with images generated from different view angles. This is of great importance when examining the anatomical structures with specular surface echoes such as tendons, nerves, and muscles because one of the several view angles will be perpendicular to the examined structure generating a higher echo amplitude, even at an insonation angle that would cause anisotropy on conventional B-mode US. ${ }^{3}$

\section{What Do We Need to Know?}

In addition to knowledge of the complex anatomy and pathologic conditions of the musculoskeletal system, the examiner must be familiar with the basic principles of US including the frequent imaging artifacts that can mimic pathology while examining normal structures and those artifacts that may accompany pathologic conditions at grayscale or Doppler imaging. ${ }^{1}$ In this article, we discuss common artifacts seen in musculoskeletal US and the techniques to avoid or minimize these artifacts during clinical US.

\section{US Features of Normal and Injured Soft Tissues}

Before discussing US imaging artifacts, we remind you briefly of the US appearance of normal and injured musculoskeletal structures. Normal muscles are hypoechoic and contain hypoechoic muscle bundles separated by fine hyperechoic fibroadipose septa (perimysium). Normal tendons are hyperechoic with a fibrillar echotexture. Normal nerves are generally hypoechoic, when compared with tendons, with speckled (or honeycomb) cross-sectional appearance (e.g., median nerve in the carpal tunnel), but they may have a relatively hyperechoic appearance in some anatomical locations relative to the adjacent musculature (e.g., median nerve in the forearm). Normal ligaments have a fibrillar or echogenic appearance. Normal cortical bone is hyperechoic with posterior acoustic shadowing. Normal epidermis is hyperechoic, whereas the hypodermis is hypoechoic and contains hyperechoic fibrous septa. In general, injured musculoskeletal tissues demonstrate discontinuity or decreased echogenicity at the site of injury when compared with normal tissues. ${ }^{1}$

\section{Artifacts in Musculoskeletal US}

On US, artifacts may cause visualization of a nonexisting structure on the displayed image or nonvisualization of an

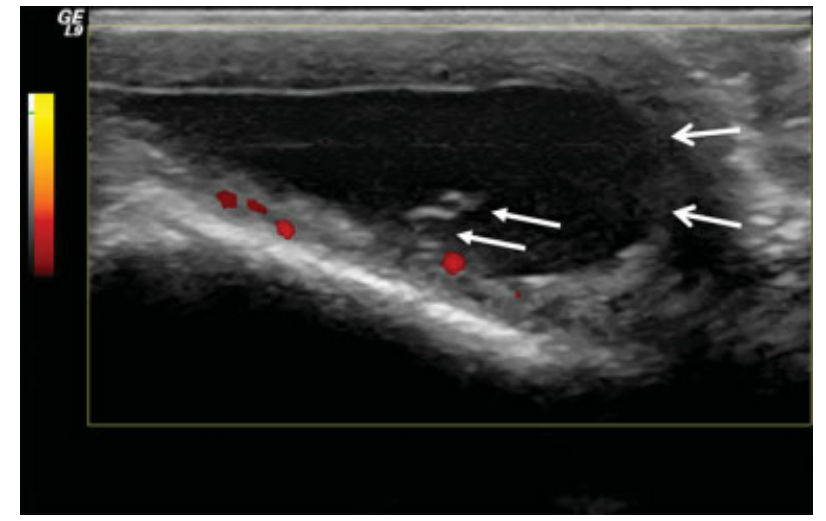

Fig. 1 Beam-width artifact in the olecranon bursa. Long-axis power Doppler ultrasound image of the posterior aspect of the olecranon shows spurious echoes within the distended olecranon bursa (solid head arrows) and reduced contrast at the lesion border (arrows) related to beam-width artifact. Additional low-level (faint) echoes within the bursa may be related to side-lobe artifact or internal debris.

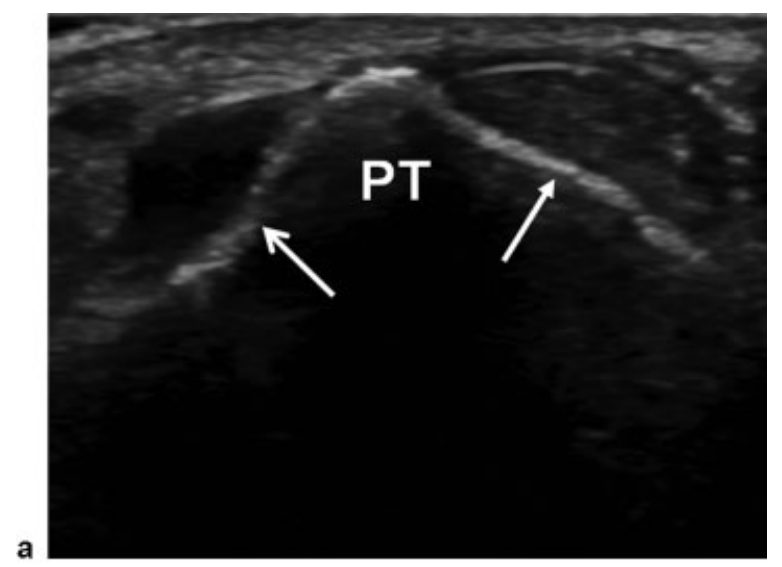

a

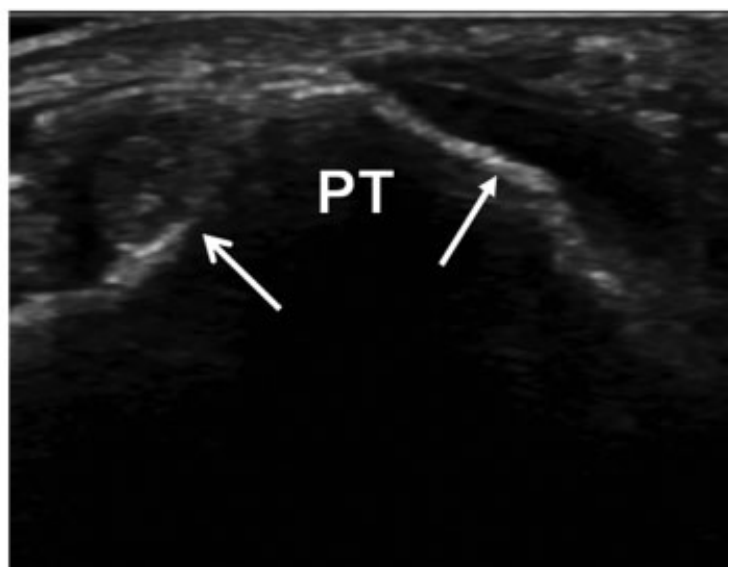

Fig. 2 Anisotropy of the peroneal tendons. Short-axis high-resolution ultrasound (US) images of the peroneal tendons in the region of the peroneal tubercle (PT) show an abnormal hypoechoic appearance of the (a) peroneus brevis (arrow) and (b) peroneus longus (solid head arrow) tendons due to anisotropy that can mimic tendinopathy and/or tendon tear. This artifact occurs when the US beam is not perpendicular to the examined anatomical structure. Note normal echogenic appearance of the (a) peroneus longus (solid head arrow) and (b) peroneus brevis (arrow) tendons in the same anatomical location with perpendicular positioning of the US transducer/beam with respect to the examined structure. 
existing structure. US artifacts may also display the existing structures in an incorrect location, size, or brightness. US artifacts may be related to US beam characteristics (side lobe and beam width, anisotropy), velocity errors (refraction), attenuation errors (posterior acoustic shadowing and increased through transmission/posterior acoustic enhancement), or they may be associated with multiple echoes (posterior reverberation, ring down, comet tail, mirror image). ${ }^{10}$

\section{Side-Lobe Artifact}

The US beam exits the transducer as a complex three-dimensional bow-tie shape with additional off-axis low-energy beams that are termed as side lobes and grating lobes. ${ }^{10}$ The main US beam narrows as it approaches the focal zone and then diverges. Side lobes are multiple beams of low-amplitude US energy that project radially from the main beam axis, which is seen mainly with linear transducers. Although the echoes generated by side lobes originate outside of the main beam, they appear as faint, specular, or diffuse artifactual echoes within the beam. ${ }^{11}$ As with beam-width artifact, this phenomenon is most likely to be recognized as extraneous echoes present within an expected anechoic structure such as the bladder, ${ }^{10}$ simple cyst, or simple fluid-filled bursa. The same phenomenon may cause the appearance of multiple needle paths during US-guided biopsy. ${ }^{12}$

\section{Beam-Width Artifact}

Beam-width artifact occurs when the US beam is too wide with respect to the imaged structure. For example, when imaging a small calcification, a relatively large beam may eliminate posterior acoustic shadowing. Additionally, if the beam is too wide, one portion of the beam may be interacting

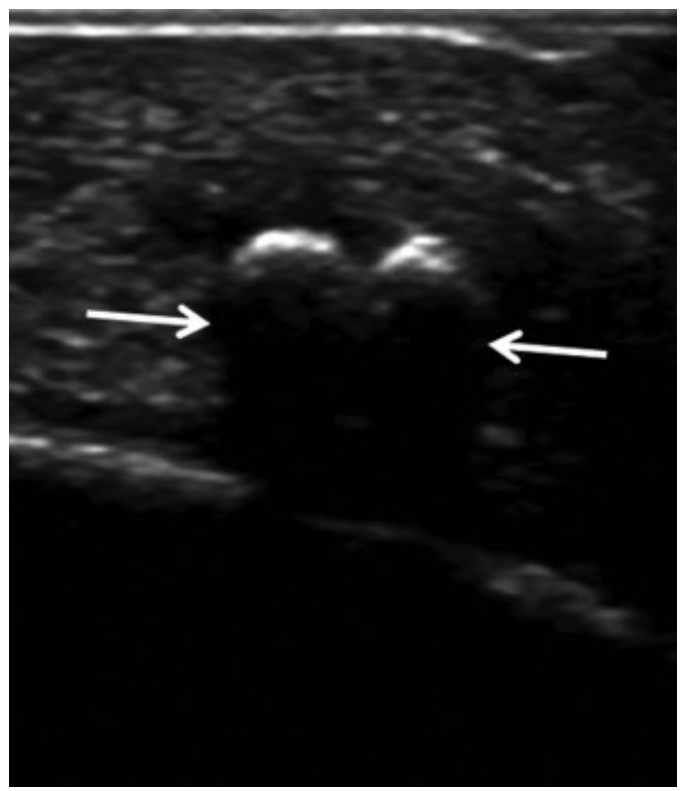

Fig. 3 Posterior acoustic shadowing: "clean" shadow. Short-axis ultrasound image of the Achilles tendon shows "clean" shadowing deep to the intratendinous calcifications that have a small radius of curvature (arrows).

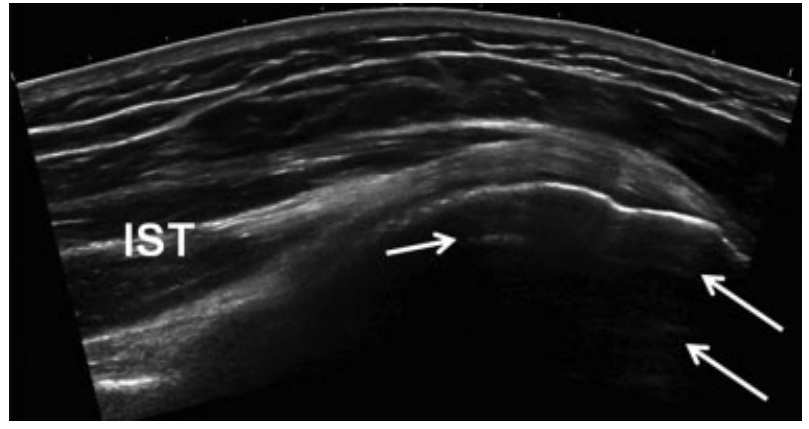

Fig. 4 Posterior acoustic shadowing: “dirty" shadow. Long-axis ultrasound image of the posterior aspect of the glenohumeral joint shows "dirty" shadowing deep to the humeral head cortex (arrows) that has a large radius of curvature (arrows). Note the infraspinatus muscle/tendon (IST).

with a fluid-filled structure while another portion of the beam interacts with adjacent soft tissues that may create spurious echoes within the cystic structures and reduced contrast at the lesion borders ( - Fig. 1). This artifact can be reduced by adjusting the focal zone at the level of the region of

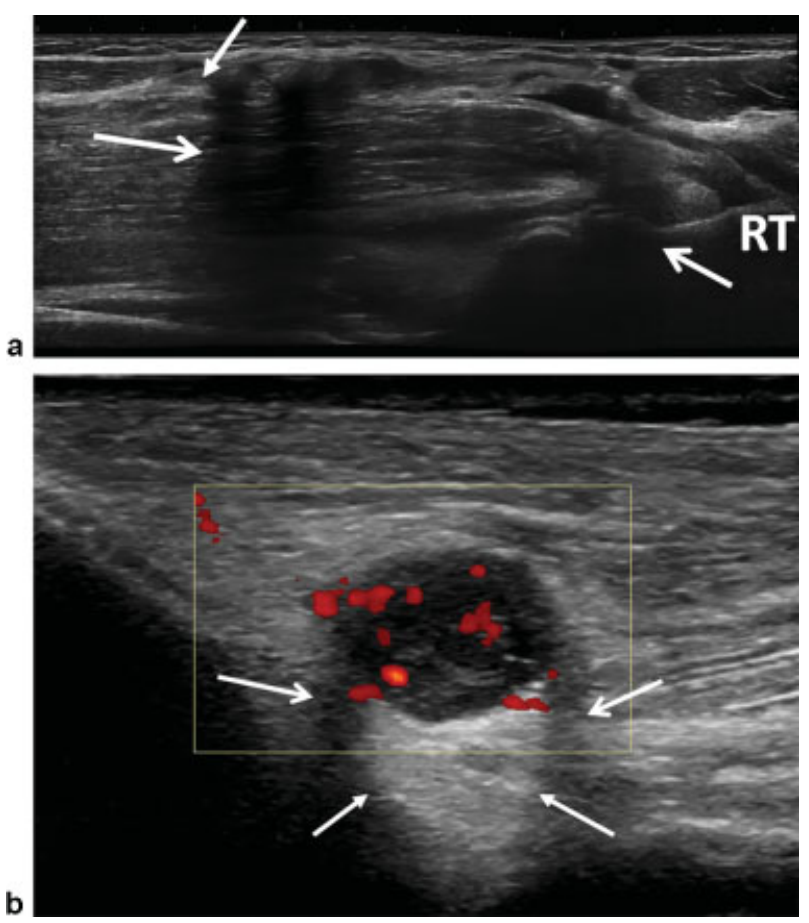

Fig. 5 Refractile shadowing (critical angle shadowing). (a) Long-axis extended field-of-view gray-scale ultrasound (US) image of an elbow shows a retracted full-thickness distal biceps tendon tear (solid head arrow) with associated lack of echoes (refractile shadowing) at and deep to the edges of the torn tendon (arrows). RT, radial tuberosity. (b) Short-axis power Doppler image of the posterolateral aspect of a knee shows a lobulated hypoechoic vascular soft tissue mass consistent with a peripheral nerve sheath tumor of the common peroneal nerve. Note lack of echoes (arrows) deep to the lateral borders of the lesion consistent with refractile shadowing and posterior acoustic enhancement (increased through transmission) deep to the lesion (solid head white arrows). 
interest or placing the transducer at the center of the examined structure. ${ }^{10,12}$

\section{Anisotropy}

Anisotropy is an US imaging artifact that appears as decreased echogenicity of the examined soft tissue when the US beam is not directed perpendicular to the examined anatomical structure. This artifact is more frequent and pronounced in tendons and ligaments due to their oblique course, and it can mimic pathology such as tendinopathy or tear. Anisotropy is less pronounced in muscles and other soft tissues, and this artifact may be used to the imager's advantage to initially identify an anatomical structure that is surrounded by the other anatomical structures of similar echogenicity (e.g., the anterior talofibular ligament). Once the anatomical location of the examined structure is identified, the transducer should be angulated perpendicular to the region of interest to eliminate this artifact. To overcome anisotropy, simple heeltoe maneuvering with manual tilting/angulation of the transducer may be used ( - Fig. 2). ${ }^{1}$ Alternatively, the beam steering feature that tilts and angulates the US beam to steer the entire B-mode image may be used, if available on the machine. ${ }^{3}$

\section{Artifacts Related to Velocity Errors}

Sound travels through soft tissues at $\sim 1540$ milliseconds (ms), bone at $4080 \mathrm{~ms}$, fat at $1450 \mathrm{~ms}$, and air at $330 \mathrm{~ms}^{13}$ If an echo travels through the examined structure with slower velocity than through the soft tissues, a longer time will be needed for that echo to return back to the transducer. The delayed return of the echo will manifest as the imaged structure being displayed deeper than its true anatomical depth. This is termed a speed displacement artifact. ${ }^{10}$

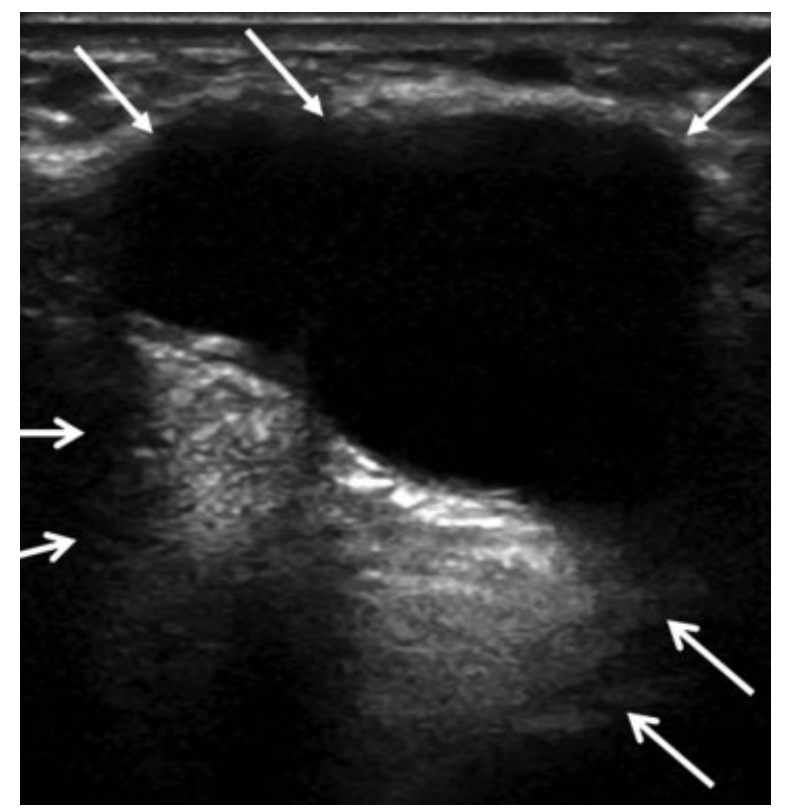

Fig. 6 Increased through transmission/posterior acoustic enhancement. Long-axis gray-scale ultrasound image of a hindfoot shows an anechoic mass consistent with a ganglion cyst (solid white head arrows) with associated increased through transmission/posterior acoustic enhancement (arrows).
Refraction represents a change in the direction of US waves as they pass from one medium to another and is accompanied by a change in speed and wavelength in media of different densities and elastic properties. In refraction, nonperpendicular incident US energy encounters an interface between two materials with different speeds of sound. When this occurs, the incident US beam changes direction. In clinical imaging, refraction artifact may cause examined structures to appear wider or misplaced, or it may cause an artifactual duplication of the structure. ${ }^{10}$

\section{Posterior Acoustic Shadowing}

Posterior acoustic shadowing is an artifact that occurs when the US beam is reflected, absorbed, or refracted, ${ }^{12}$ and it appears as an anechoic area that extends deep from the involved interface. Posterior acoustic shadowing occurs at
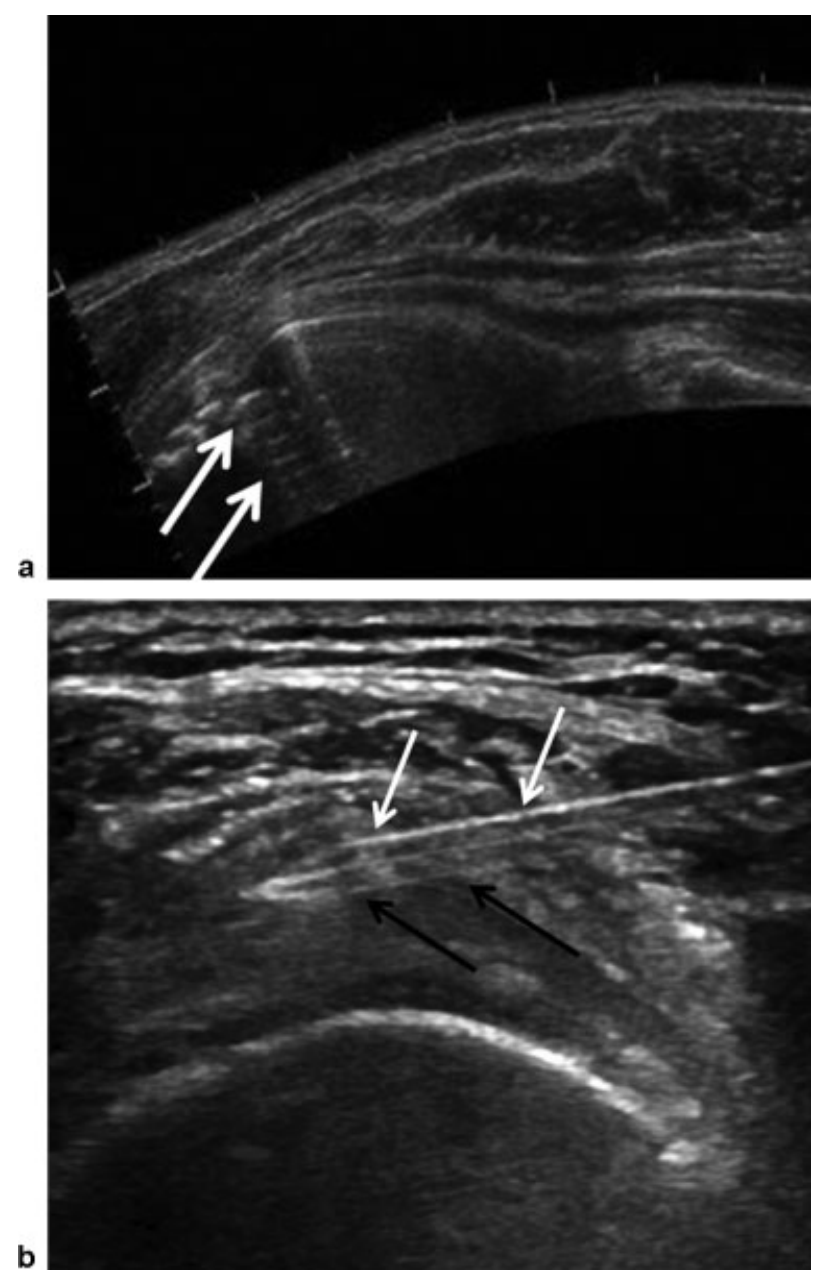

Fig. 7 Posterior reverberation. (a) Long-axis extended field-of-view gray-scale ultrasound (US) image of the posterior aspect of a shoulder in a patient with shoulder arthroplasty shows series of linear reflective echoes that extend deep to the metal-bone interface spaced at equal distances consistent with posterior reverberation artifact (arrows). (b) Long-axis gray-scale US image of the supraspinatus tendon shows a calcium lavage needle in the region of the calcific tendinitis (white arrows). Note a series of linear reflective echoes deep to the needle spaced at equal distances consistent with posterior reverberation artifact (black arrows). 


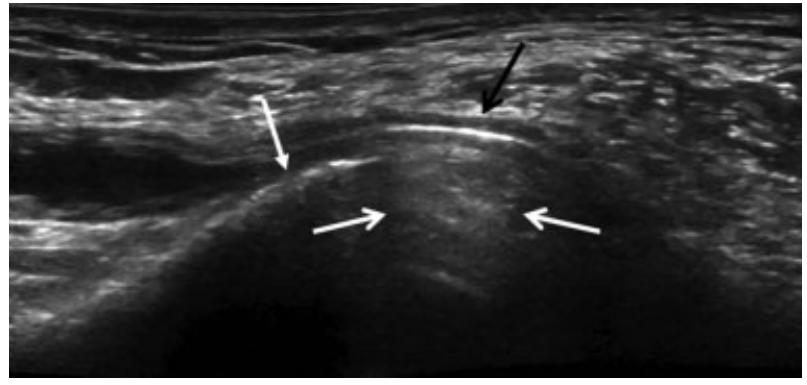

Fig. 8 Ring-down artifact. Long-axis gray scale ultrasound image at the lateral aspect of a knee in a patient with total knee arthroplasty shows a series of continuous reflective echoes (white arrows) deep to the examined highly reflective metal surface (black arrow) consistent with a ring-down artifact. Note distal femoral cortex (solid head white arrow) with associated posterior acoustic shadowing.

interfaces with bone or calcification, some foreign bodies, and gas. ${ }^{1}$ Objects that have a small radius of curvature or a rough surface display a clean shadow ( $\mathbf{F i g . ~ 3 ) ; ~ t h o s e ~ w i t h ~ a ~ l a r g e ~}$ radius of curvature and a smooth surface result in a dirty shadow (-Fig. 4), represented by heterogeneous hypoechogenicity, resulting from superimposed reverberation echoes. ${ }^{14}$ Refractile shadowing (also known as critical angle shadowing) may occur at the edges of some structures, such as foreign bodies or the ends of a torn tendon ( - Fig. 5a). This artifact typically occurs because the incident beam is both reflected by the curved surface and often refracted by the different tissue type, with none of the incident sound beam striking the lateral margin returning to the transducer, resulting in a lack of echoes (shadow) deep to the lateral borders of the structure ( $\mathbf{- F i g} . \mathbf{5 b}) .{ }^{12,15}$

\section{Posterior Acoustic Enhancement/Increased through Transmission}

Posterior acoustic enhancement or increased through transmission occurs with imaging of fluid filled lesions ( - Fig. $\mathbf{6}$ ) and soft tissue tumors, such as peripheral nerve sheath

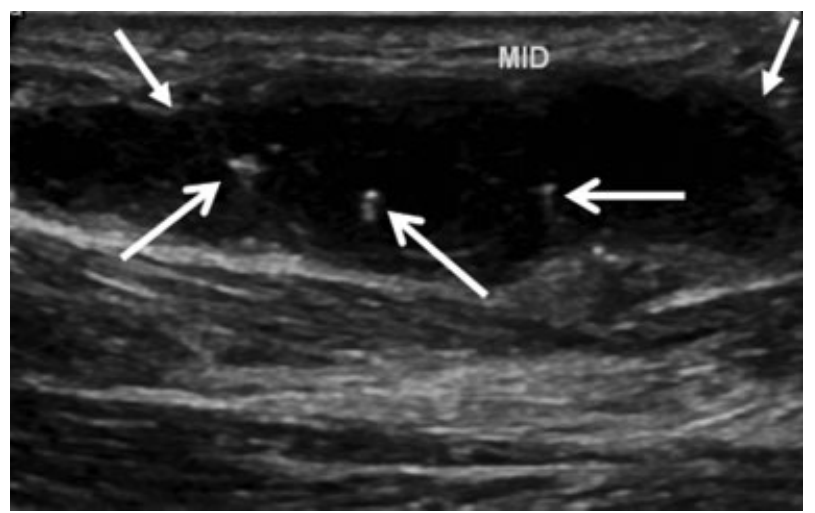

Fig. 9 Comet-tail artifact related to air in an infected soft tissue fluid collection. Long-axis gray-scale ultrasound image of the proximal forearm shows a thick-walled anechoic fluid collection in the subcutaneous soft tissues (solid head arrows). Note several dense tapering echo trails just distal to small strongly reflecting structures related to a comet-tail artifact (arrows) indicating presence of intralesional air in an infected soft tissue fluid collection.

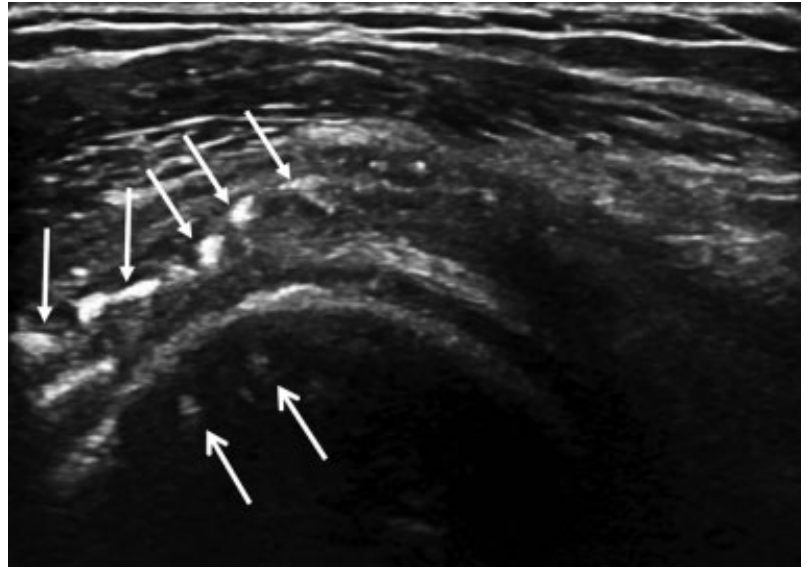

Fig. 10 Mirror image. Long-axis gray-scale ultrasound (US) image of the shoulder shows several foci of increased echogenicity (solid head arrows) in the supraspinatus tendon consistent with calcific tendinitis (calcium hydroxyapatite deposition). Note an incompletely portrayed, distorted mirror image of the supraspinatus tendon calcifications (arrows) projecting in the adjacent humeral head, which is a highly reflective acoustic interface, secondary to reverberation artifact as a result of scattering of the US waves.

tumors ( - Fig. $\mathbf{5 b}$ ) and giant cell tumor of tendon sheath. ${ }^{16}$ This artifact occurs due to relatively lower attenuation of the sound beam in the imaged lesion when compared with the adjacent soft tissues, which results in a hyperechoic appearance of soft tissues deep to the lesion. ${ }^{12}$ Presence of this artifact in solid lesions is believed to be related to homogeneity and cellularity of solid lesions. Identification of flow on color or power Doppler imaging helps to differentiate cystic lesions from nerve sheath tumors and other soft tissue masses. $^{16}$

\section{Posterior Reverberation}

When examining two parallel highly reflective surfaces, the echoes generated from a primary US beam may be repeatedly reflected back and forth before returning to the transducer, resulting in a multiecho display. This artifact is termed posterior reverberation. After a single reflection, the first echo returned to the transducer will be displayed in the proper location; the sequential echoes will take a longer time to return and will be displayed on an image at an increased distance from the transducer. In US imaging, this is termed posterior reverberation artifact and has an appearance of a series of linear reflective echoes that extend deep to the examined structure and spaced at equal distances (-Fig. 7). ${ }^{10,12}$

Ring-down artifact is a posterior reverberation artifact seen with scanning metal surfaces and has an appearance of a series of more continuous reflective echoes deep to the examined metal surface ( $\mathbf{- F i g . ~ 8 ) .}{ }^{1}$ This artifact does not obscure any soft tissue structures that are superficial to the metal, making US an ideal imaging modality in the evaluation of peri-hardware soft issues. ${ }^{1,10}$

\section{Comet-Tail Artifact}

Comet-tail artifact appears as a dense tapering trail of echoes just distal to a strongly reflecting structure. This posterior 
reverberation type of artifact occurs when there is a marked difference in acoustic impedances between an object and the surrounding tissues. The comet-tail artifact was first described by Ziskin et al in 1982. In soft tissues, the comet tail is commonly caused by metal, plastic, or air. ${ }^{17,18}$ US is often more sensitive than radiographs for the detection of subtle soft tissue collections of air, and the comet-tail artifact may indicate the presence of a soft tissue abscess or tissue necrosis (-Fig. 9). ${ }^{10,12}$

\section{Mirror Image}

Imaged structures immediately adjacent to highly reflective acoustic interfaces may appear duplicated secondary to reverberation at their interface as a result of scattering of the US waves. On the US images, this artifact results in an inverted, duplicated, distorted, and incompletely portrayed mirror image (-Fig. 10). ${ }^{10}$

\section{US Artifacts with Doppler Imaging}

\section{Transducer Pressure}

An important avoidable artifact that occurs with Doppler imaging may be caused by an excessive amount of transducer pressure, which may block the flow in small vessels and diminish flow in larger vessels (-Fig. 11). This may be obviated by applying copious amounts of US gel, which allows for good acoustic contact and decreases pressure on examined tissues. $^{7}$
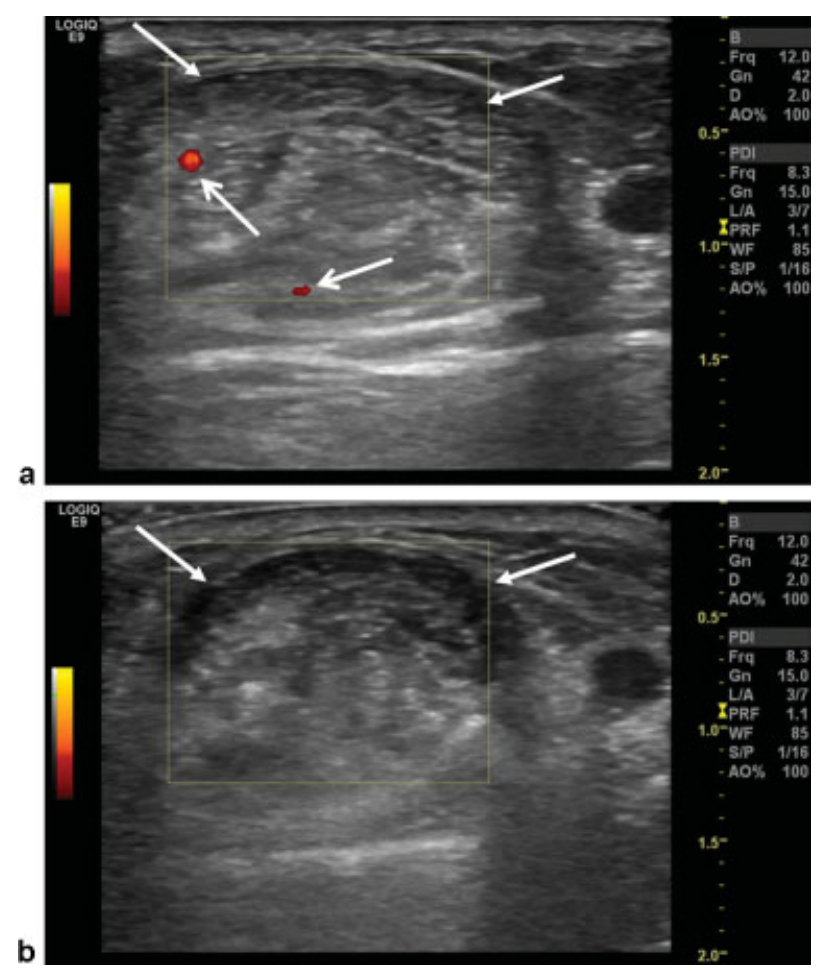

Fig. 11 Transducer pressure. (a) Power Doppler short-axis ultrasound (US) image of an Achilles tendon shows two small vessels within the tendon (arrows). (b) These vessels are not seen on subsequent shortaxis power Doppler US image of the same region after the excessive amount of transducer pressure that was applied blocked the flow.

\section{Tissue Strain}

Tissue strain that is affected by patient positioning is also important because the tension on the imaged musculoskeletal soft tissues may increase the internal pressure and diminish or remove Doppler signal. ${ }^{7}$

\section{Focus}

Care must be taken to choose the proper focus cursor inside of the image, which indicates where the pulse is narrowest and the energy most concentrated. ${ }^{7}$

\section{Motion}

Motion of the patient or slow movement of the tissue or vessel wall caused by arterial pulsation gives a motion relative to the transducer and produces a Doppler shift. ${ }^{19}$ Motion of the transducer will produce the same effect. These movements produce lower frequency Doppler shifts ${ }^{20}$ that appear as random short flashes of confluent color. ${ }^{7}$ This type of motion artifact may be avoided by using high-pass wall filters, but these will also remove the information from slow flow moving blood. ${ }^{20}$

\section{Blooming Artifact}

Blooming artifact displays color outside of a vessel and makes vessels appear larger (-Fig. 12). ${ }^{21}$ This is usually caused by the gain setting, which can be adjusted. Decreasing the Doppler gain minimizes the blooming artifact, but the weakest signal from the smallest vessels may be undetected. ${ }^{7}$

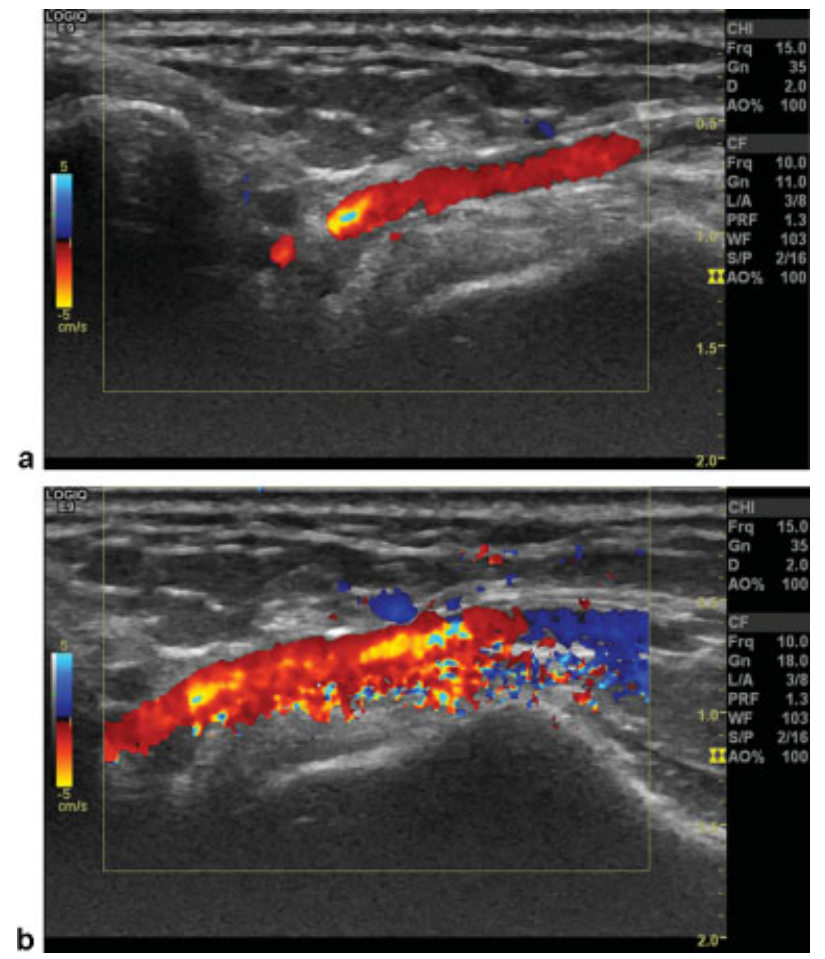

Fig. 12 Blooming artifact. (a) Color Doppler long-axis ultrasound image of a radial artery proximal to the radiocarpal joint shows normal caliber radial artery with associated small amount of aliasing (foci of green color within the artery). (b) Subsequent increased color Doppler gain in the same region caused artificial enlargement of a vessel with color displayed outside of the radial artery. Note increased aliased flow in reversed colors. 


\section{Mirror Image}

Reflection of Doppler signal from highly reflective bone surfaces may generate a false mirror image inside the bone of a vessel that is adjacent to bone, due to the reflected signal. ${ }^{19,21,22}$ The main problems that may arise using color Doppler for imaging the musculoskeletal system that are not encountered with power Doppler are background noise, aliasing, and more pronounced angle dependency. ${ }^{5}$

\section{Background Noise}

Color Doppler has a random direction of flow on the image because noise has a random frequency shift (-Fig. 13a). Power Doppler, in contrast, has a uniformly low noise. Increasing the gain on color Doppler increases the noise
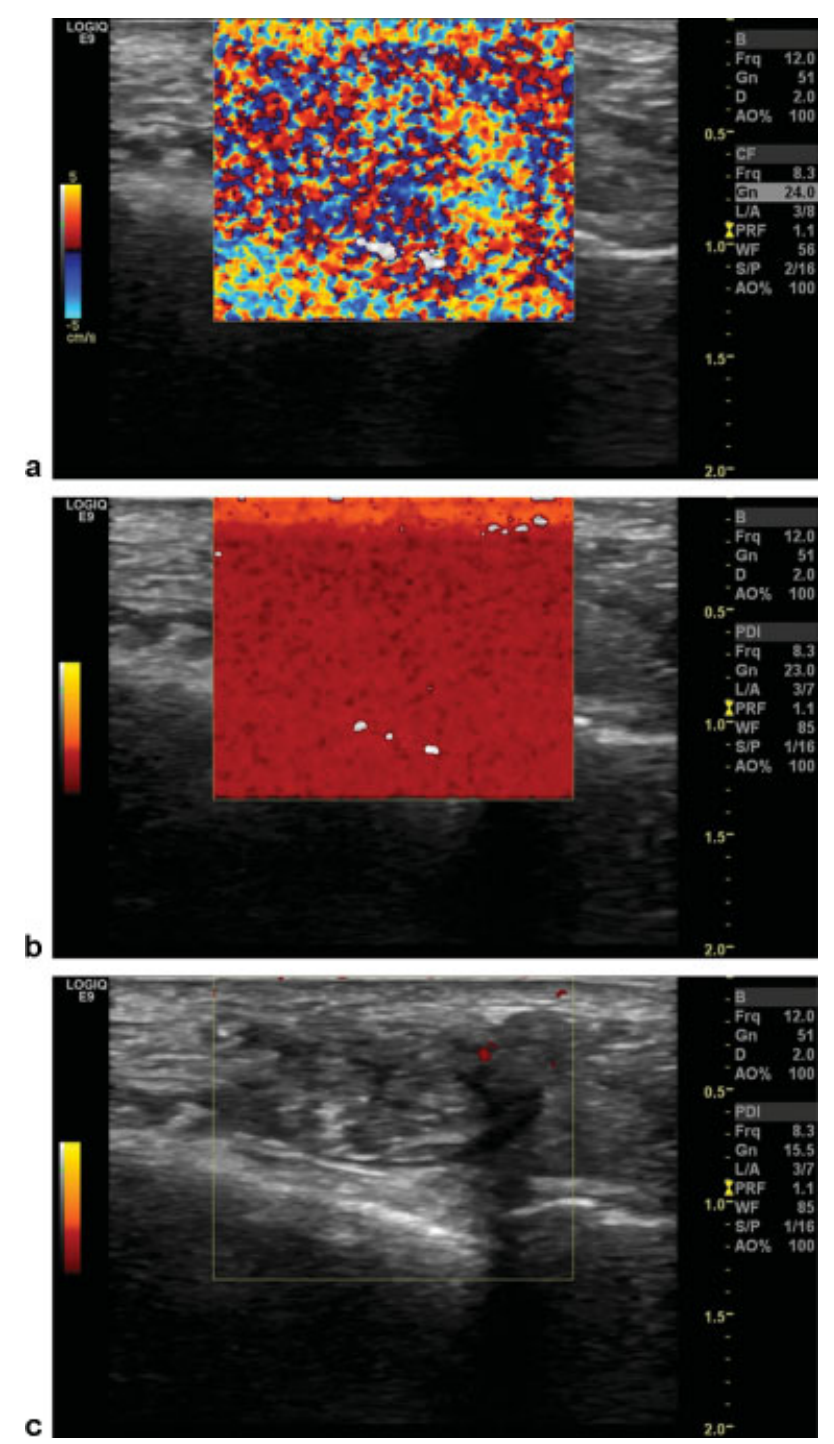

Fig. 13 Background noise. (a) Increased gain on color Doppler longaxis ultrasound image along the lateral aspect of the calcaneus increased the noise and degraded the image. Note a random direction of flow (reversed colors) in the Doppler box because the noise has a random frequency shift. (b) Increased power Doppler gain in the same region caused a uniformly colored background in the Doppler box with an enhanced signal from true flow because the power Doppler has a uniformly low noise. (c) The power Doppler gain setting was then properly decreased until almost all noise disappeared. and thereby the image is degraded. When power Doppler gain is increased, there is a uniformly colored background with an enhanced signal from true flow (-Fig. 13b). The sensitivity and dynamic range of power Doppler is therefore greater than color Doppler. ${ }^{5}$ To achieve maximum sensitivity, the gain setting should be increased until noise pixels dominate the Doppler box and then decreased until almost all noise has disappeared (- Fig. 13b, c) ${ }^{7}$

\section{Aliasing}

Aliasing is the most common artifact in Doppler US, seen both in color and spectral Doppler modalities. It reflects the point at which the maximum measurable velocity has been reached for a given pulse repetition frequency and gain setting. If the Doppler shift frequency exceeds half of pulse repetition frequency/Nyquist limit (normal is $1-30 \mathrm{KHz}$ range), aliasing occurs and improper Doppler shift information (improper direction and improper value) results. Aliasing in a color flow system is exposed in a spatial 2D plane in which the aliased flow is shown in reversed color surrounded by nonaliased flow (-Fig. 14a). ${ }^{5,7}$ If aliasing is present on the screen, the overall gain should be decreased to the point immediately before aliasing appears; if this does not satisfactorily reduce aliasing, the pulse repetition frequency may be adjusted/ increased and the Doppler angle increased to improve the image. However, by increasing the pulse repetition frequency, the sensitivity for low flow is decreased. ${ }^{7}$ The color maps for

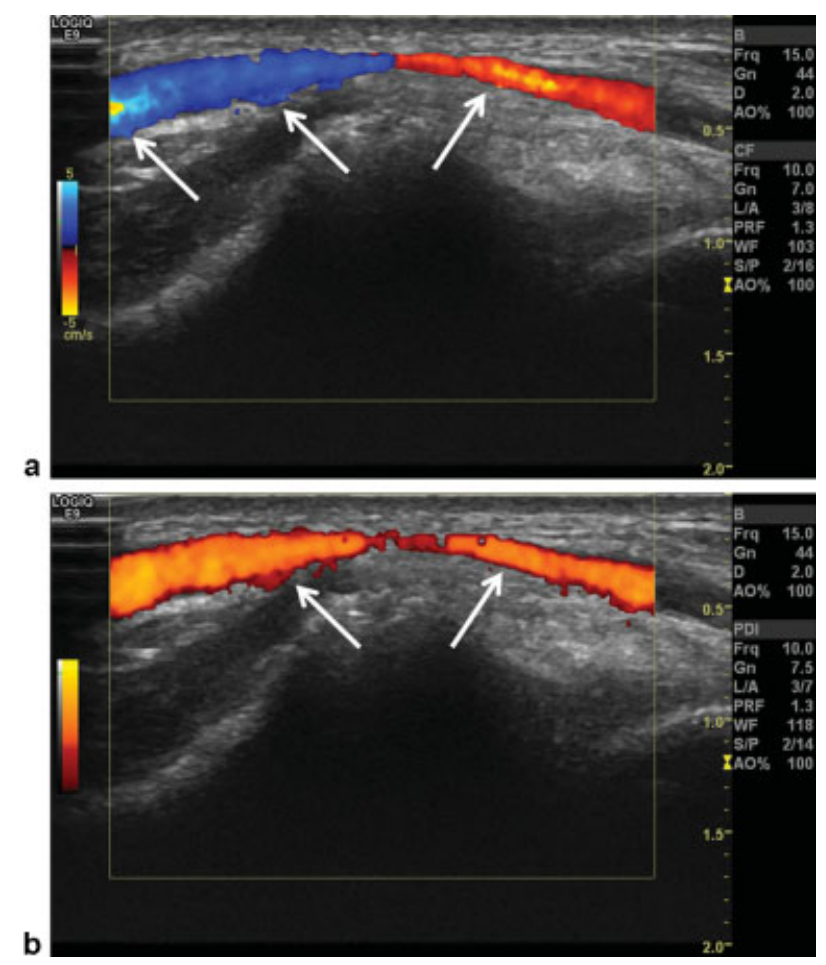

Fig. 14 Aliasing artifact. (a) Long-axis color Doppler ultrasound image of a radial artery at the level of the radiocarpal joint shows reversed blue color (aliased flow) and red color (nonaliased flow) that was eliminated in (b). (b) Power Doppler image in the same region shows a uniformly red-colored radial artery. Because power Doppler does not provide directional flow information, the aliasing artifact did not occur. 


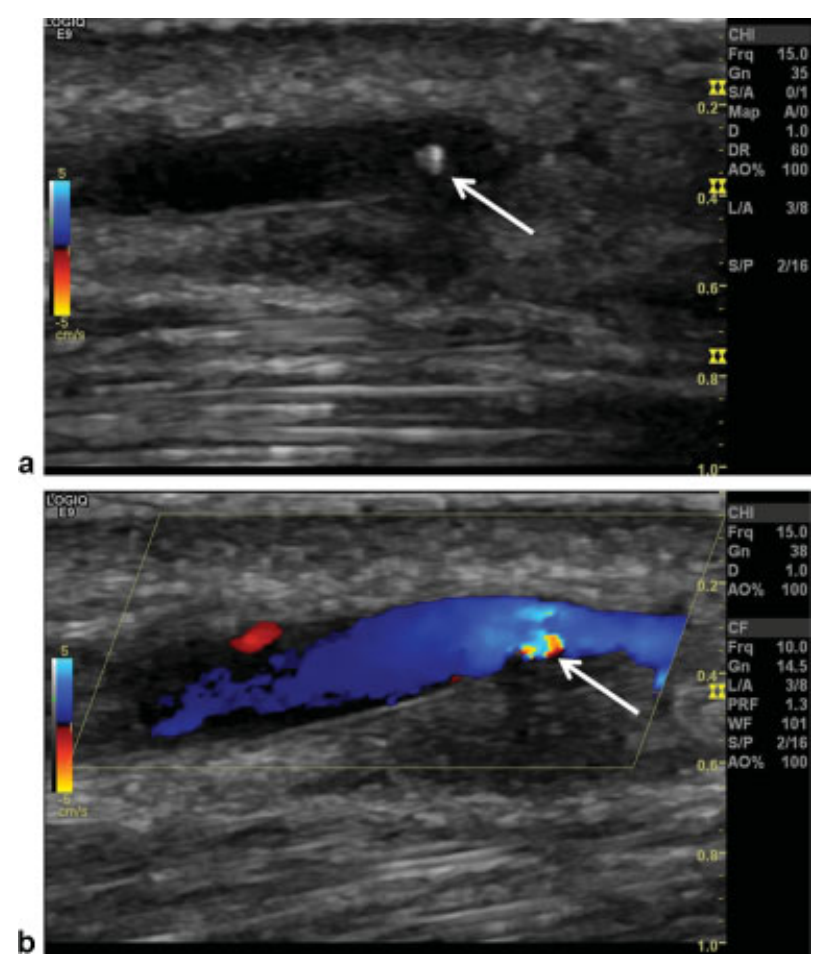

Fig. 15 Twinkling artifact. (a) Color Doppler long-axis ultrasound image of a forearm shows a focus of increased echogenicity within the basilic vein consistent with a protruding cactus thorn (arrow). (b) On the subsequent image with a Doppler box placed in the region of interest, note color signal in the region of the cactus thorn (arrow) without associated real flow related to a twinkling artifact.

power Doppler are represented by a single continuous color (hot color map). Because power Doppler does not provide directional flow information, the aliasing artifact does not $\operatorname{occur}\left(-\right.$ Fig. 14b) ${ }^{5}$

\section{Twinkling Artifact}

Twinkling artifact is a color phenomenon seen behind a strongly reflecting interface such as urinary tract stones, parenchymal calcifications, bones, or foreign bodies during color Doppler US examination. This artifact appears as a color signal but without associated real flow. It imitates turbulent flow (-Fig. 15). ${ }^{23}$ The appearance of the twinkling artifact is highly machine and setting dependent. The underlying cause is likely a narrow bandwidth noise introduced by phase (or clock) jitter in the Doppler circuitry of the US scanner. ${ }^{24}$

\section{Conclusion}

Imaging artifacts are common with musculoskeletal US. Some of them can mimic pathology, and some occur with pathologic conditions and may help in diagnosis. When performing and interpreting musculoskeletal US, the radiologist has to be able to recognize these artifacts and know how to eliminate or minimize them to improve diagnostic quality.

\section{References}

1 Jacobson JA. Fundamentals of Musculoskeletal Ultrasound. 2nd ed. Philadelphia, PA: Elsevier Saunders; 2012

2 Jacobson JA. Musculoskeletal ultrasound: focused impact on MRI. AJR Am J Roentgenol 2009;193(3):619-627

3 Klauser AS, Peetrons P. Developments in musculoskeletal ultrasound and clinical applications. Skeletal Radiol 2009; September 3 (Epub ahead of print)

4 Smith J, Finnoff JT. Diagnostic and interventional musculoskeletal ultrasound: part 1. Fundamentals. PM R 2009;1(1):64-75

5 Teh J. Applications of Doppler imaging in the musculoskeletal system. Curr Probl Diagn Radiol 2006;35(1):22-34

6 Hedrick WR, Hykes DL, Starchman DE. Doppler physics and instrumentation. In: Ultrasound Physics and Instrumentation. 4th ed. St. Louis, MO: Mosby; 2005

7 Boesen MI, Boesen M, Langberg H, et al. Musculoskeletal colour/ power Doppler in sports medicine: image parameters, artefacts, image interpretation and therapy. Clin Exp Rheumatol 2010;28(1): 103-113

8 Rubin JM, Bude RO, Carson PL, Bree RL, Adler RS. Power Doppler US: a potentially useful alternative to mean frequency-based color Doppler US. Radiology 1994;190(3):853-856

9 Taljanovic MS, Goldberg MR, Sheppard JE, Rogers LF. US of the intrinsic and extrinsic wrist ligaments and triangular fibrocartilage complex-normal anatomy and imaging technique. Radiographics 2011;31(1):e4410.1148/rg.e44

10 Feldman MK, Katyal S, Blackwood MS. US artifacts. Radiographics 2009;29(4):1179-1189

11 Laing FC, Kurtz AB. The importance of ultrasonic side-lobe artifacts. Radiology 1982;145(3):763-768

12 Scanlan KA. Sonographic artifacts and their origins. AJR Am J Roentgenol 1991;156(6):1267-1272

13 Bushberg JT, Seibert JA, Leidholdt EM, Boone JM. The Essential Physics of Medical Imaging. 3rd ed. Baltimore, MD: Lippincott Williams \& Wilkins; 2011

14 Rubin JM, Adler RS, Bude RO, Fowlkes JB, Carson PL. Clean and dirty shadowing at US: a reappraisal. Radiology 1991;181(1):231-236

15 Hartgerink P, Fessell DP, Jacobson JA, van Holsbeeck MT. Fullversus partial-thickness Achilles tendon tears: sonographic accuracy and characterization in 26 cases with surgical correlation. Radiology 2001;220(2):406-412

16 Reynolds DL Jr, Jacobson JA, Inampudi P, Jamadar DA, Ebrahim FS, Hayes CW. Sonographic characteristics of peripheral nerve sheath tumors. AJR Am J Roentgenol 2004;182(3):741-744

17 Ziskin MC, Thickman DI, Goldenberg NJ, Lapayowker MS, Becker JM. The comet tail artifact. J Ultrasound Med 1982;1(1):1-7

18 Thickman DI, Ziskin MC, Goldenberg NJ, Linder BE. Clinical manifestations of the comet tail artifact. J Ultrasound Med 1983;2(5): 225-230

19 Pozniak MA, Zagzebski JA, Scanlan KA. Spectral and color Doppler artifacts. Radiographics 1992;12(1):35-44

20 Rubin JM. Spectral Doppler US. Radiographics 1994;14(1):139-150

21 Nilsson A. Artefacts in sonography and Doppler. Eur Radiol 2001; 11(8):1308-1315

22 Arning C. Mirror image artifacts of color Doppler images causing misinterpretation in carotid artery stenoses. J Ultrasound Med 1998;17(11):683-686

23 Rahmouni A, Bargoin R, Herment A, Bargoin N, Vasile N. Color Doppler twinkling artifact in hyperechoic regions. Radiology 1996;199(1):269-271

24 Kamaya A, Tuthill T, Rubin JM. Twinkling artifact on color Doppler sonography: dependence on machine parameters and underlying cause. [published correction appears in AJR Am J Roentgenol 2003;180:542]. AJR Am J Roentgenol 2003;180(1):215-222 